\title{
Design and Realization of Position Controller Based on Characteristic Model for Servo Systems with Large Inertia Ratio
}

\author{
Zhihong Wang ${ }^{1, *}$, Wei Chen ${ }^{2}$, Yifei $\mathrm{Wu}^{1}$, Xiang Wang ${ }^{1}$, Jian Guo $^{1}$ and Qingwei Chen ${ }^{1}$ \\ ${ }^{1}$ School of Automation, Nanjing University of Science and Technology, Nanjing 210094, China \\ ${ }^{2}$ Electronic and Mechanical Technology Department of the Nanjing Marine Radar Institute, Nanjing 210003, China
}

\begin{abstract}
An adaptive sliding mode controller (ASMC) based on characteristic model is designed to overcome the detrimental effect of large inertia ratio and large-range varying inertia in high accuracy servo systems. The servo system discrete characteristic model is established and adopted for the controller designed instead of using the traditional mechanism model. The recursive least square (RLS) algorithm is used to identify time-varying parameters in characteristic model. The position controller is constituted by an adaptive equivalent controller based on identification parameters and an improved sliding mode controller, and the stability of the closed-loop system is analyzed. The experimental results show that the proposed controller can adapt to large-range varying inertia, and improve the dynamic performance and steady-state precision of servo systems.
\end{abstract}

Keywords: Large inertia ratio, Servo systems, Characteristic model, Adaptive sliding mode control.

\section{INTRODUCTION}

Servo systems have been widely used in various applications, such as weapon systems, radars, machine tools, robots and astronomical telescopes. With the rapid development of national defense and national economy, the requests for high precision, good dynamic performance and good adaptability have been proposed to servo systems. For instance, a suppressing multiple launch rocket system cannot be regarded as a rigid body, because the long barrel structure makes it flexible. In the launching process, the load inertia and torque varies in a large range due to varying bomb load. What's more, the nonlinearities such as uncertain friction are present in the system which makes it more difficult for traditional control algorithms to meet the control requirements.

Considering the large range variation of system parameters and disturbance torque, high speed and high precision control algorithms with good adaptability and disturbance rejection ability are required. Existing control methods includes sliding mode control [1-3], Kalman filter prediction control [4], neural networks control [5], wavelet based sliding mode control $[6,7]$, disturbance observer $[8,9]$ etc. In [2], an adaptive backstepping sliding mode controller was proposed for pitch position control system of the rocket launcher, backstepping control was adopted to guarantee the stability of the closed-loop tracking system, and sliding mode control was adopted to restrain the parameter perturbation and external disturbances. In [5], a sliding mode adaptive controller was designed based on neural network, and

*Address correspondence to this author at the School of Automation, Nanjing University of Science and Technology, Nanjing 210094, China;

E-mail: njust.wang@gmail.com neural network was applied to replace the switching function in sliding mode control. In [6], fuzzy wavelet neural network was used to approximate the equivalent control variable and a terminal sliding mode position controller was designed for high power weapon servo systems. In [10], an adaptive controller based on extended state observer and inertia estimation was developed for servo systems with variable inertia. Existing methods can achieve good control performance when system parameters or load inertia varies within a small range, but there are still many deficiencies such as dependence of precise mathematical model, multiple adjustable parameters, complex debugging process, difficult determination of parameter range and slow convergence of the adaptive parameters. It is also difficult to achieve good control performance when the system parameters vary in a large range.

In our research, the characteristic model is adopted to describe the servo systems instead of the traditional mechanism model [10] or other modeling methods of different fields [11-13], which are fairly complicated or not suitable for servo systems compared with the characteristic model. Characteristic modeling is a new modeling method proposed by $\mathrm{Wu}$ $[14,15]$. The system satisfying certain conditions can be equivalent with a second-order linear time-variant system which makes it easier to design the controller, and the parameters can be determined beforehand within a fairly small range, which is beneficial to fast parameter convergence when estimating the parameters online with the RLS algorithm. The nonlinear components and system parameter changes are also considered in the characteristic model which makes the designed controller with better performance and robustness. In sliding mode control, the system states are forced to run on the sliding-mode surface, and it has good 


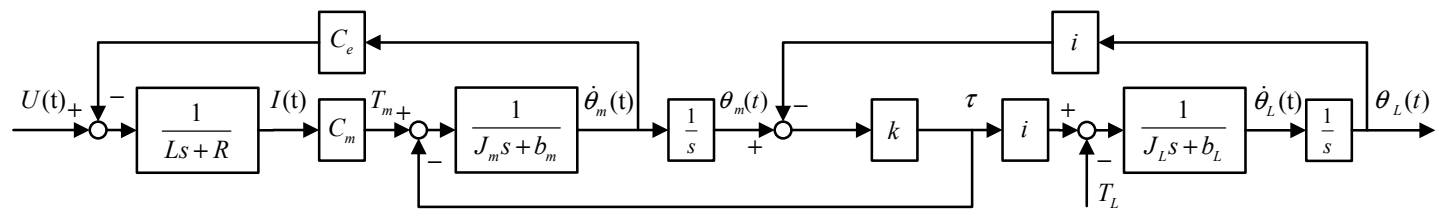

Fig. (1). Structure diagram of the servo system.

disturbance rejection ability and robustness to uncertainties and external disturbances [16].

In this paper, characteristic modeling and sliding mode control are combined together. The system with large variation of load inertia is described with characteristic model, and the model parameters are identified online, which brings good adaptability to the controller. On the other hand, the using of sliding mode control can also bring good robustness to the controller. As a result, an adaptive sliding mode controller is proposed and the simulation and experimental results show that the proposed method can obtain good performance with varying inertia, and has good adaptive ability and robustness.

The reminder of this paper is organized as follows. Section 2 gives the original dynamic model and the characteristic model of the servo system. In Section 3, an adaptive sliding mode controller based on characteristic model is proposed, and the stability of the closed-loop system is analyzed. Section 4 and Section 5 present the simulation and experimental results respectively. Finally, the conclusion is given in Section 6.

\section{PROBLEM DESCRIPTION AND SYSTEM MODEL- ING}

The dynamic equations of a typical electromechanical system driven by gears can be expressed as

$$
\left\{\begin{array}{l}
U(t)=C_{e} \dot{\theta}_{m}(t)+R I(t)+L \dot{I}(t) \\
J_{m} \ddot{\theta}_{m}(t)+b_{m} \dot{\theta}_{m}(t)=T_{m}-\tau \\
J_{L} \ddot{\theta}_{L}(t)+b_{L} \dot{\theta}_{L}(t)=i \tau-T_{L} \\
T_{m}=C_{m} I(t) \\
\tau=k\left(\theta_{m}-i \theta_{L}\right)
\end{array}\right.
$$

where $U(t), I(t), R, L, C_{e}$ and $C_{m}$ denote the armature voltage, armature current, the resistance, the inductance, the back electromotive force (EMF) coefficient and the torque coefficient of the motor respectively. $\theta_{m}, \dot{\theta}_{m}, \ddot{\theta}_{m}, J_{m}, b_{m}$, $\theta_{L}, \dot{\theta}_{L}, \ddot{\theta}_{L}, J_{L}$ and $b_{L}$ denote the angular displacement, the angular velocity, the angular acceleration, the rotational inertia, the viscous friction coefficient of the motor and the load respectively. $T_{m}, T_{L}, \tau, k$, and $i$ denote the motor torque, the load torque, the elastic torque between the motor and the load, the stiffness coefficient and the gear ratio respectively. The structure of the system is shown in Fig. (1).

The nonlinear system is expressed as $\dot{x}(t)=f\left(x, \dot{x}, \cdots, x^{(n)}, u, \dot{u}, \cdots u^{(m)}\right)$

Define $\quad x=x_{1}, \dot{x}=x_{2}, \cdots, x^{(n)}=x_{n+1}$ and $u=u_{1}, \dot{u}=u_{2}, \cdots, u^{(m)}=u_{m+1}$, then (2) can be rewritten as $\dot{x}(t)=f\left(x_{1} \cdots x_{n+1}, u_{1} \cdots u_{m+1}\right)$

The nonlinear system (3) is assumed as tem.

1) The system is a single input single output (SISO) sys-

2) The order of the control input $u(t)$ is 1 .

3) $f(\cdot)=0$ when all the bounded variables $x_{i}$ and $u_{i}$ equal to zero.

4) $f(\cdot)$ is continuous differentiable to all the variables $x_{i}$ and $u_{i}$, and all partial derivatives are bounded.

5) $|f(x(t+\Delta t), u(t+\Delta t))|-|f(x(t), u(t))|<M \Delta t$, where $\Delta t$ is the sample period, and $M$ is a positive constant.

The assumption (1), (2) and (3) are easily satisfied for most servo systems, and considering the nonlinearities in servo systems, such as the friction, the nonlinear component could be described by a continuous differentiable approximate function to make the model smooth, and satisfies the assumption (4). The assumption (5) can also be satisfied by using a relatively small value of sample time $\Delta t$.

Lemma 1: (see [15]): If the system (3) satisfies the above assumptions, the characteristic model of the system can be established by a second-order slow time-varying difference equation as (4).

$$
\begin{aligned}
& x(k+1)=f_{1}(k) x(k)+f_{2}(k) x(k-1)+g_{0}(k) u(k) \\
& +g_{1}(k) u(k-1)
\end{aligned}
$$

where $f_{1}(k), f_{2}(k), g_{0}(k)$ and $g_{1}(k)$ are the time-varying parameters of the model, which can be estimated online. And the parameter range can be determined as $f_{1}(k) \in(1,2]$, $f_{2}(k) \in[-1,0), g_{0}(k)<<1$ and $g_{1}(k) \ll<1$, which has been proved in [15].

If system (3) is a minimum phase system, the $g_{1}(k) u(k-1)$ in equation (4) can be neglected. The characteristic model of the system can be written as

$x(k+1)=f_{1}(k) x(k)+f_{2}(k) x(k-1)+g_{0}(k) u(k)$ 
In [15], a new parameter estimation algorithm is proposed to estimate the time-varying parameters with less operation, but the convergence of the algorithm is relatively slow and the estimation value is sensitive to the initial value of the parameters. As a result, the new estimation algorithm can only be used in some particular conditions. Therefore the RLS algorithm with forgetting factor is adopted to estimate the time-varying parameters $f_{1}(k), f_{2}(k)$ and $g_{0}(k)$ online owning to the fast convergence rate and insensitivity to the initial value of the parameters.

In our research, we also found that a properly selected initial value of $f_{1}(k), f_{2}(k)$ and $g_{0}(k)$ is benefit for a fast convergence of the RLS algorithm. In the experiments, we selected the average value of the upper and lower bound for $f_{1}(k)$ and $f_{2}(k)$ as the initial value of the parameters without using all zeros.

\section{CONTROLLER DESIGN AND STABILITY ANAL- YSIS}

The error characteristic model of the system can be expressed as a second-order slow time-varying difference equation:

$e(k+1)=f_{1}(k) e(k)+f_{2}(k) e(k-1)+g_{0}(k) u(k)$

The RLS is applied to estimate the time-varying parameters $f_{1}(k), f_{2}(k)$ and $g_{0}(k)$ online as $\hat{f}_{1}(k), \hat{f}_{2}(k)$ and $\hat{g}_{0}(k)$, then the identification model is obtained as

$e(k+1)=\hat{f}_{1}(k) e(k)+\hat{f}_{2}(k) e(k-1)+\hat{g}_{0}(k) u(k)+\Delta(k)$

Where $\Delta(k)$ denote the sum of model identification error, uncertainty and external disturbance. Assuming $|\Delta(k)|<f_{m}$, where $f_{m}$ is a known positive constant, which is reasonable for most servo systems.

The control action is designed as follows:

$u(k)=u_{1}(k)+u_{2}(k)$

Where $u_{1}(k), u_{2}(k)$ are the adaptive equivalent control law and the sliding mode control law respectively, which satisfy

$u_{1}(k)=-\frac{1}{\hat{g}_{0}}\left[\hat{f}_{1}(k) e(k)+\hat{f}_{2}(k) e(k-1)\right]$

$u_{2}(k)=\frac{1}{\hat{g}_{0}}\{(1-q T) e(k)-\varepsilon T|e(k)| \arctan [e(k)]\}$

Where $T$ denote the sample period, $q, \varepsilon$ and $T$ are constants satisfying

$q>0, \varepsilon>\frac{f_{m}}{T\left|e_{0}(k)\right| \arctan \left|e_{0}(k)\right|}, 0<T<\frac{1}{q+\pi \varepsilon}$
Where $e_{0}(k)$ denote the designed minimal tracking error.

Theorem1: If the controller (8) is applied to system (7), and the parameters satisfy the condition (9), then the closedloop system is stable.

Proof: Take (8) into (7) to obtain

$e(k+1)=(1-q T) e(k)-\varepsilon T|e(k)| \arctan [e(k)]+\Delta(k)$

Such that

$[e(k+1)-e(k)] \operatorname{sign}[e(k)]$

$=\{-q T e(k)-\varepsilon T|e(k)| \arctan [e(k)]+\Delta(k)\} \operatorname{sign}[e(k)]$

$=-q T|e(k)|-\varepsilon T|e(k)| \arctan |e(k)|+\Delta(k) \operatorname{sign}[e(k)]$

$<-q T|e(k)|-\varepsilon T|e(k)| \arctan |e(k)|+f_{m}$

$<-\varepsilon T|e(k)| \arctan |e(k)|+f_{m}$

$<-\frac{f_{m}}{\left|e_{0}(k)\right| \arctan \left|e_{0}(k)\right|}|e(k)| \arctan |e(k)|+f_{m}$

$\leq 0$

i.e.

$[e(k+1)-e(k)] \operatorname{sign}[e(k)]<0$

$e(k+1) e(k)$

$=[(1-q T) e(k)-\varepsilon T|e(k)| \arctan [e(k)]+\Delta(k)] e(k)$

$=(1-q T) e^{2}(k)-\varepsilon T e^{2}(k) \arctan |e(k)|+\Delta(k) e(k)$

According to $0<\arctan |e(k)|<\frac{\pi}{2}$, we obtain

$e(k+1) e(k)$

$>\left(1-q T-\frac{\pi}{2} \varepsilon T\right) e^{2}(k)+\Delta(k) e(k)$

$=(1-q T-\pi \varepsilon T) e^{2}(k)+\frac{\pi}{2} \varepsilon T e^{2}(k)+\Delta(k) e(k)$

Considering $\varepsilon>\frac{f_{m}}{T\left|e_{0}(k)\right| \arctan \left|e_{0}(k)\right|}>\frac{2 f_{m}}{\pi T\left|e_{0}(k)\right|}$, we obtain

$$
\begin{aligned}
& (1-q T-\pi \varepsilon T) e^{2}(k)+\frac{\pi}{2} \varepsilon T e^{2}(k)+\Delta(k) e(k) \\
& >(1-q T-\pi \varepsilon T) e^{2}(k)+\frac{f_{m} e^{2}(k)}{\left|e_{0}(k)\right|}+\Delta(k) e(k) \\
& =(1-q T-\pi \varepsilon T) e^{2}(k)+|e(k)|\left\{f_{m} \frac{|e(k)|}{\left|e_{0}(k)\right|}+\Delta(k) \operatorname{sign}[e(k)]\right\} \\
& \geq(1-q T-\pi \varepsilon T) e^{2}(k)+|e(k)|\left[f_{m} \frac{\left|e_{0}(k)\right|}{\left|e_{0}(k)\right|}-f_{m}\right] \\
& =(1-q T-\pi \varepsilon T) e^{2}(k)
\end{aligned}
$$

According to (9), we get $1-q T-\pi \varepsilon T>0$ 
Then we obtain $e(k+1) e(k)>0$, i.e.

$[e(k+1)+e(k)] e(k)>0,[e(k+1)+e(k)] \operatorname{sign}[e(k)]>0$

The verification of two conditions $[e(k+1)-e(k)] \operatorname{sign}[e(k)]<0 \quad$ and $[e(k+1)+e(k)] \operatorname{sign}[e(k)]>0$ proves that the closed-loop system is stable.。

Theorem 1 gives the range of parameters which guarantee the stability of the closed-loop system. In the proof process, using $e(k+1) e(k)>0$ instead of $[e(k+1)+e(k)] \operatorname{sign}[e(k)]>0$ in traditional discrete sliding mode control can ensure the sign of the error is constant in the convergence process, and make the error converge to a small neighborhood of the origin. The following part will further analysis the convergence range of the error.

Theorem 2: If the controller (8) is applied to system (7), and the parameters satisfy the condition (9), then

$\left|e_{0}(k)\right|<\frac{-q T+\sqrt{q^{2} T^{2}+4 \varepsilon T f_{m}}}{2 \varepsilon T}$

Proof:

According to $e(k+1) e(k)>0$, we can obtain that $e(k+1)$ and $e(k)$ have the same sign, define $\lambda=\frac{e(k+1)}{e(k)}$

Then we have $\lambda=1-q T-\varepsilon T \arctan |e(k)|+\frac{\Delta(k)}{e(k)}$

When $\lambda=1$, the tracking error converges to a fairly small value $e_{0}(k)$, we get

$-q T-\varepsilon T \arctan \left|e_{0}(k)\right|+\frac{\Delta(k)}{e_{0}(k)}=0$, i.e.

$\Delta(k)=q T e_{0}(k)+\varepsilon T e_{0}(k) \arctan \left|e_{0}(k)\right|$

Assuming $e_{0}(k)$ converges to a small neighborhood of the origin, and $e_{0}(k)$ is small enough to obtain $\arctan \left[e_{0}(k)\right] \approx e_{0}(k)$.

Take $\arctan \left[e_{0}(k)\right]=e_{0}(k)$ into $(10)$ to obtain :

$\Delta(k)=q T e_{0}(k)+\varepsilon T e_{0}(k)\left|e_{0}(k)\right|$

According to the assumption $|\Delta(k)|<f_{m}$, we get

$\left|q T e_{0}(k)+\varepsilon T e_{0}(k)\right| e_{0}(k)||<f_{m}$

To analyze equation (11), we must check the following two conditions:

1. when $e_{0}(k)>0$

Equation (11) becomes: $\varepsilon T e_{0}^{2}(k)+q T e_{0}(k)-f_{m}<0$

Then we obtain:

$\frac{-q T-\sqrt{q^{2} T^{2}+4 \varepsilon T f_{m}}}{2 \varepsilon T}<e_{0}(k)<\frac{-q T+\sqrt{q^{2} T^{2}+4 \varepsilon T f_{m}}}{2 \varepsilon T}$

Considering $e_{0}(k)>0$, we obtain:

$0<e_{0}(k)<\frac{-q T+\sqrt{q^{2} T^{2}+4 \varepsilon T f_{m}}}{2 \varepsilon T}$

2. When $e_{0}(k)<0$

Equation (11) becomes:

$\varepsilon T e_{0}^{2}(k)-q T e_{0}(k)-f_{m}<0$

Then we obtain:

$\frac{q T-\sqrt{q^{2} T^{2}+4 \varepsilon T f_{m}}}{2 \varepsilon T}<e_{0}(k)<\frac{q T+\sqrt{q^{2} T^{2}+4 \varepsilon T f_{m}}}{2 \varepsilon T}$

Considering $e_{0}(k)<0$, we obtain:

$$
-\frac{-q T+\sqrt{q^{2} T^{2}+4 \varepsilon T f_{m}}}{2 \varepsilon T}<e_{0}(k)<0
$$

According to (12) and (13), the convergence range of $e_{0}(k)$ is obtain as:

$$
\left|e_{0}(k)\right|<\frac{-q T+\sqrt{q^{2} T^{2}+4 \varepsilon T f_{m}}}{2 \varepsilon T}
$$

\section{SIMULATION RESULTS}

For the controller design of servo systems, the Matlab/Simulink is the most widely used simulation tool, and the other feasible alternatives such as ADAMS is also used in some particular applications. ADAMS is usually used to make dynamic analysis and simulation of mechanical systems, and the model-based controller design is relatively weak compared with Simulink. Therefore, in order to demonstrate the effectiveness of the proposed ASMC, simulations are firstly carried out in Matlab/Simulink in terms of step responses.

The system parameters are selected as $R=1.3 \Omega$, $L=0.0375 \mathrm{H}, C_{e}=67.2 \mathrm{~V} / \mathrm{krpm} \quad, \quad C_{m}=1.11 \mathrm{~N} \cdot \mathrm{m} / \mathrm{A}$ ， $J_{m}=0.000323 \mathrm{~kg} \cdot \mathrm{m}^{2} \quad, \quad b_{m}=0.015 \mathrm{~N} \cdot \mathrm{m} / \mathrm{krpm} \quad$, $b_{L}=0.024 \mathrm{~N} \cdot \mathrm{m} / \mathrm{krpm}, k=1.3 \times 10^{6} \mathrm{~N} \cdot \mathrm{m} / \mathrm{rad}, i=178$ and the load inertia $J_{L}$ can be $40.94,122.81$ and $204.68 \mathrm{~kg} \cdot \mathrm{m}^{2}$ which is 4,12 and 20 times of the motor inertia.

For the convenience of comparison, the control parameters of adaptive sliding mode controller and proportion integration differentiation (PID) controller are adjusted to meet the requirements and get similar performance with the load 


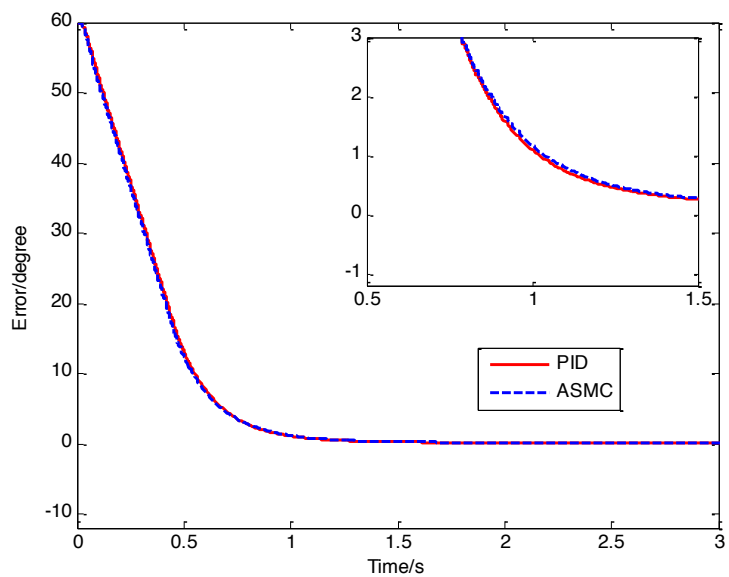

Fig. (2). $60^{\circ}$ step tracking error with load inertia ratio of $4: 1$.

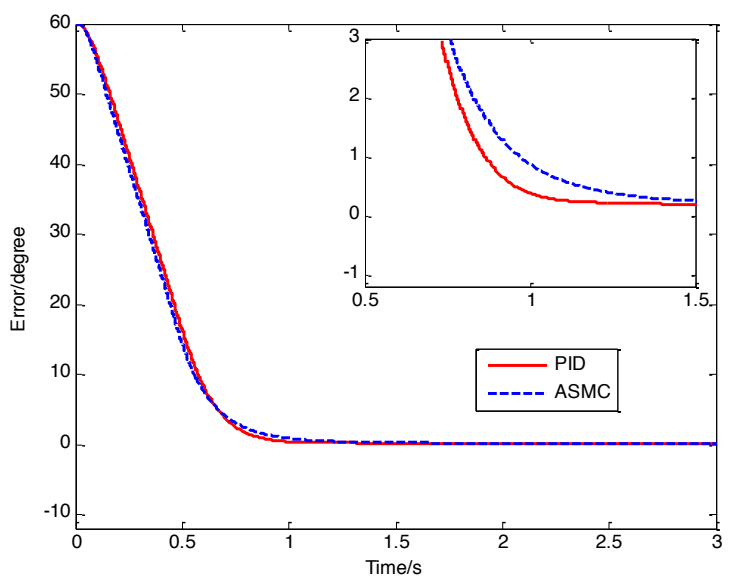

Fig. (3). $60^{\circ}$ step tracking error with load inertia ratio of 12:1.

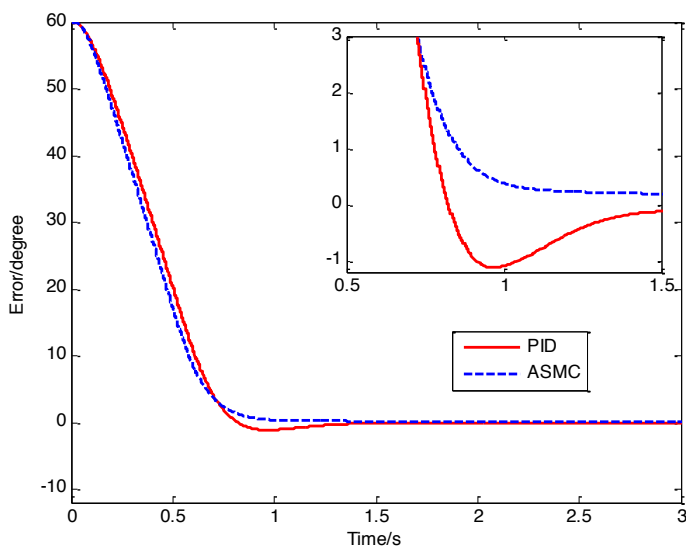

Fig. (4). $60^{\circ}$ step tracking error with load inertia ratio of 20:1.

inertia ratio of $4: 1$. Then, all the controller parameters are fixed, and the control performances of the two controllers are compared with the load inertia ratio of $12: 1$ and 20:1 respectively.

The parameters of adaptive sliding mode controller are selected as $q=82, \varepsilon=23$, and $T=0.005$, the RLS forget- ting factor is $f=0.995$, and the parameters of PID controller are $K_{p}=407, K_{i}=25$.

The responses of $60^{\circ}$ step command with different load inertias are tested. The tracking errors are shown in Fig. (2) to Fig. (4). 
Table 1. Parameters of the motor.

\begin{tabular}{|c|c|c|c|}
\hline Name & Units & B-402-B & M-403-B \\
\hline \hline Rated power & $\mathrm{kW}$ & 2.2 & 3000 \\
\hline Rated speed & $\mathrm{RPM}$ & 3000 & 0.00259 \\
\hline Inertia & $\mathrm{Kg} \cdot \mathrm{m}^{2}$ & 0.000323 & 0.24 \\
\hline Static friction & $\mathrm{N} \cdot \mathrm{m}$ & 0.24 & 0.015 \\
\hline Viscous friction & $\mathrm{N} \cdot \mathrm{m} / \mathrm{kRPM}$ & 0.015 & 0 \\
\hline
\end{tabular}

Table 2. Parameters of the reducer.

\begin{tabular}{|c|c|c|}
\hline Name & Units & F1C-A35-89 \\
\hline \hline Reduction ratio & & 69 \\
\hline Rated output torque & $\mathrm{N} \cdot \mathrm{m}$ & 638 \\
\hline Input shaft Inertia & $\mathrm{Kg} \cdot \mathrm{m}^{2}$ & 0.000433 \\
\hline Max input speed & RPM & 3950 \\
\hline
\end{tabular}

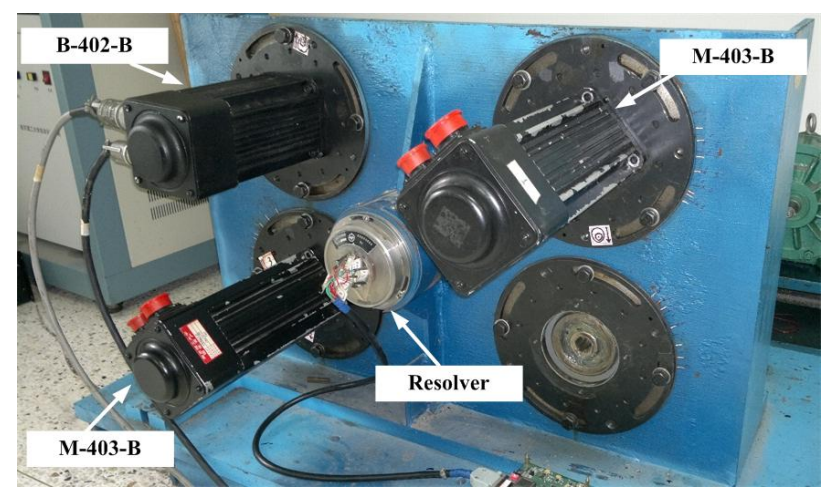

Fig. (5). The motor side of experimental system.

As shown in Fig. (2, 3 and 4), the control performance of the PID controller is affected greatly by the variation of load inertia. In Fig. (2), the performance of PID controller is almost the same as the proposed controller with load inertia ratio of 4:1. With the increasing of load inertia ratio, the performance of PID controller has gradually become poor, and obvious overshoot appears with load inertia ratio of 20:1. On the other hand, the dynamic response of the proposed controller changes much less than that of the PID controller owning to the adaptability of characteristic model and the robustness of the proposed controller. The simulation results have proved that the proposed controller can provide an adaptive and robust control performance under load inertia variation.

\section{EXPERIMENTAL RESULTS}

To practically evaluate the actual performance of the proposed ASMC, it is necessary to compare it with the traditional PID controller through the position loop tracking experiments carried out on the experimental system. The sampling time of position loop is $5 \mathrm{~ms}$. Two types of permanent magnet synchronous motor (PMSM) produced by Kollmorgen and the reducer produced by Sumitomo Heavy Machinery Company are utilized in the experiment. The parameters of the motor and the reducer are listed in Table $\mathbf{1}$ and Table 2.

According to Table 1, the inertia of the motor M-403-B is 8 times that of the motor B-402-B, and other parameters are the same. The reduction ratio of the reducer is $1: 89$, and the reduction ratio between the large and small gears is $1: 2$, therefore the inertia of the large and small gears converted to motor side is relatively small, and it can be ignored. According to Table 1 and Table 2, the inertia of the experimental bench with four reducers is 4 times that of the motor B-402$\mathrm{B}$, therefore, when the experimental bench is driven by one motor B-402-B, the load inertia ratio is $4: 1$. Then one or two M-403-B motors can be installed to simulate different load inertia ratio of 12:1 or 20:1, as shown in Fig. $(\mathbf{5 , 6})$. 


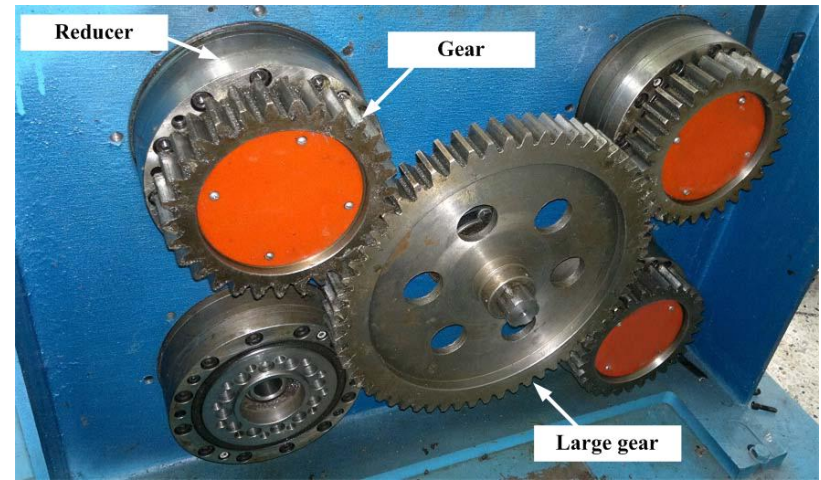

Fig. (6). The load side of experimental system.

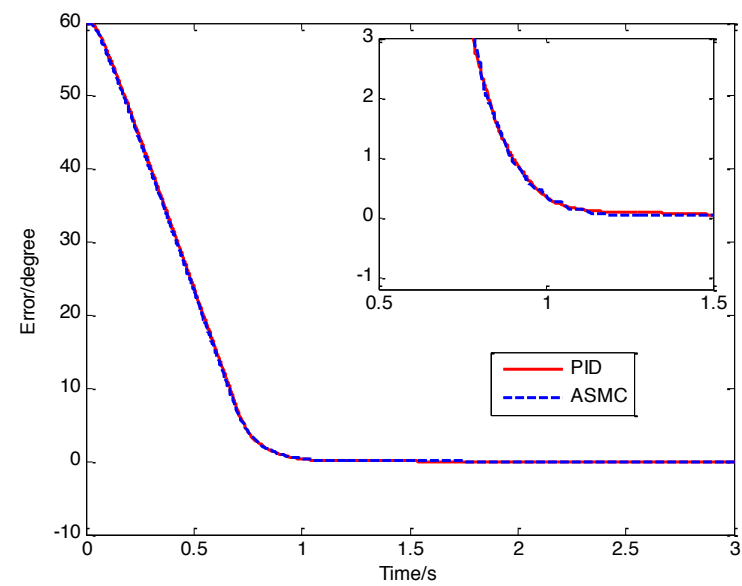

Fig. (7). $60^{\circ}$ step tracking error with load inertia ratio of $4: 1$.

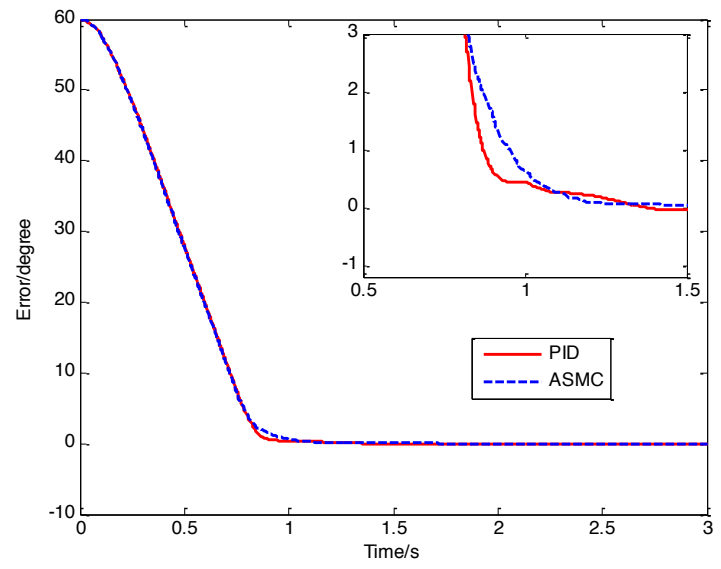

Fig. (8). $60^{\circ}$ step tracking error with load inertia ratio of $12: 1$.

It is similar to Section 4 that the control parameters of ASMC and PID controller are adjusted to get similar performance with the load inertia ratio of $4: 1$. Then, all the controller parameters are fixed, and the control performances of the two controllers are compared with the load inertia ratio of $12: 1$ and $20: 1$ respectively.

After experimental adjustment, the parameters of ASMC are selected as $q=10, \varepsilon=5$, the RLS forgetting factor is $f=0.995$, and the parameters of PID controller are
$K_{p}=5.0, K_{i}=0.1, K_{d}=20$. The responses of $60^{\circ}$ step command with different load inertias are tested. The tracking errors are shown in Fig. (7) to Fig. (9), and the identification results of the characteristic model parameters are shown in Fig. (10).

According to the experimental results shown in Figs. (79), when the load inertia ratio increases with fixed controller parameters, obvious overshoot appears with the PID 


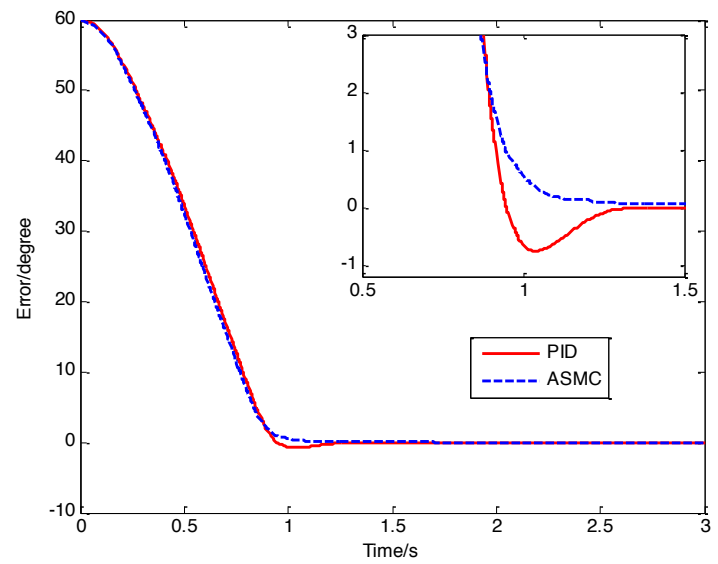

Fig. (9). $60^{\circ}$ step tracking error with load inertia ratio of 20:1.
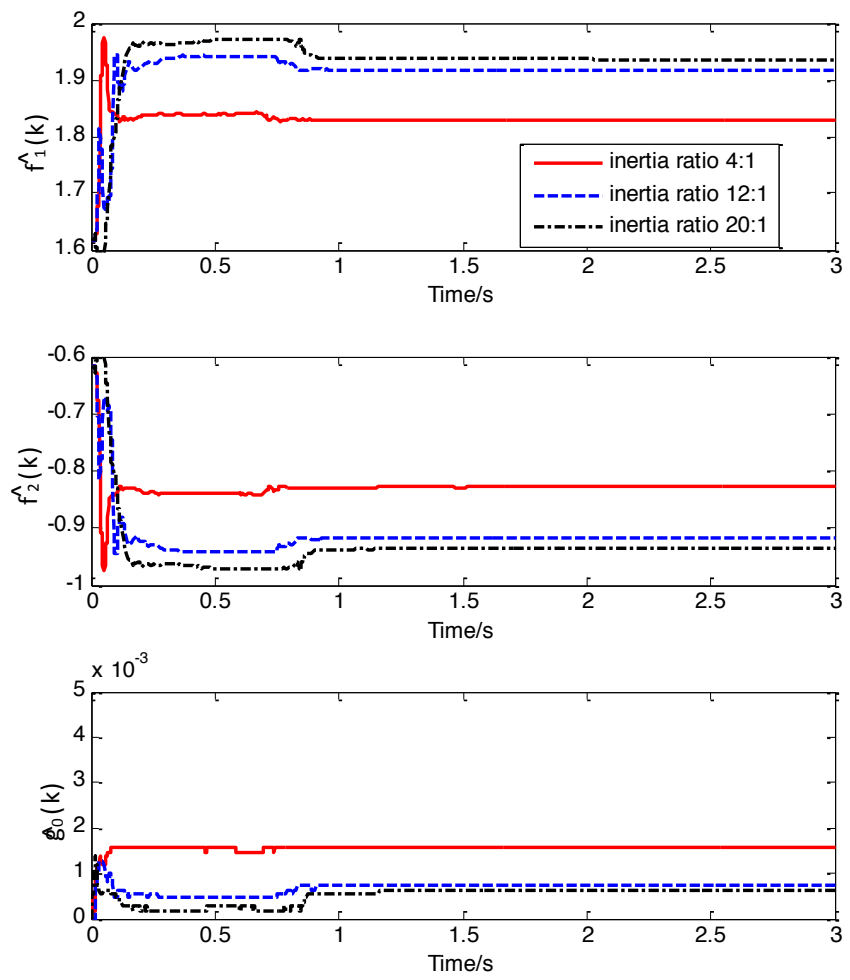

Fig. (10). identification results of model parameters with different load inertia.

controller, and the control performance is poor due to the variation of load inertia. On the other hand, as discussed in Section 2, the parameters of characteristic model can be determined beforehand within a small range, which makes it easier to achieve fast convergence. As shown in Fig. (10), the parameters of characteristic model is estimated online, and the identification values of model parameters vary with different load inertia, which reflects the strong adaptability of characteristic model, as a result, the proposed adaptive sliding mode controller can still keep good tracking performance with large-range varying inertia due to the online identification of the characteristic model and the robustness of the sliding mode control, and has better adaptability and robustness compared with the PID controller.

\section{CONCLUSION}

This paper has proposed an adaptive sliding mode controller based on the characteristic model for high speed and high precision servo systems with large inertia ratio and large-range varying inertia. The characteristic model of the system is established based on the characteristic modeling method, and parameters of the model are obtained online by the RLS algorithm with forgetting factor. The chattering phenomenon of traditional sliding mode control is eliminated by an improved form of controller. The closed-loop stability of the system has been proved by Lyapunov theory. It is shown by both simulation and experimental results that the proposed controller can give better performance with 
large-range varying inertia compared with traditional PID controller, and has better adaptability and robustness. This work can also be improved by relaxing the application conditions of characteristic model and the designed controller to make it easier to use and suitable for more different applications.

\section{CONFLICT OF INTEREST}

The authors confirm that this article content has no conflicts of interest.

\section{ACKNOWLEDGEMENTS}

This work was supported by the National Natural Science Foundation of China under Grant nos. 61074023, Science and Technology Support Program of Jiangsu Province under Grant No. BE2012175 and "333 Project" Foundation of Jiangsu Province under Grant No. BRA2012163.

\section{REFERENCES}

[1] L.Yi, G. Hong, Y. Yongqiang, "Sliding mode control of permanent magnet synchronous motor with great inertia load", Journal of Beijing University of Aeronautics and Astronautics, vol.33, no. 10, pp. 1208-1211, 2007.

[2] G. Ya-jun, W. Xiao-feng, M.A. Da-wei, and L.E. Gui-gao, "Application of adaptive backstepping sliding mode control in alternative current servo system of rocket gun", Acta Armamentarii, vol.32, no. 4, pp.493-497, 2011.

[3] F. Peihua, C. Zhen, C. Binglong, and Z. Jing," A position servo system of permanent magnet synchronous motor based on backstepping adaptive sliding mode control", Transactions Of China Electrotechnical Society, vol. 28, no. 9, pp. 288-293, 2013.

[4] W. Zheng-jun, and Z. Jiang-bo, "Large inertia servo system predictive brake control based on dual Kalman filter" Electric Machines and Control, vol. 15, no. 8, pp. 75-80, 2011.
[5] Y. Yongqiang, G. Hong, and X. Zhanming, "Neural network adaptive sliding mode control for direct-drive servo system", Transactions of China Electrotechnical Society, vol. 24, no. 3, pp. 74-79, 2009.

[6] H. Run-min, Li. Rong-zhong, H. Yuan-Long, G. Qiang, and Y. Guo-lai, "Application of adaptive fuzzy wavelet neural sliding mode control in ac servo system", Acta Armamentarii, vol. 35, no. 6, pp. 769-775, 2014.

[7] L.W. Lee, and I.H. Li, "Wavelet-based adaptive sliding-mode control with $\mathrm{H} \infty$ tracking performance for pneumatic servo system position tracking control", IET Control Theory and Applications, vol. 6, no.11, pp. 1699-1714, 2012.

[8] L. Wen, and H. Yoichi, "Vibration suppression using single neuron-based pi fuzzy controller and fractional-order disturbance observer", IEEE Transactions on Industrial Electronics, vol. 54, no. 1, pp. 117-126, 2007.

[9] J. S. Bang, H. Shim, S. K. Park, and J. H. Seo, "Robust tracking and Vibration suppression for a two-inertia system by combining backstepping approach with disturbance observer", IEEE Transactions on Industrial Electronics, vol. 57, no. 9, pp. 3197-206, 2010.

[10] L. Shihua, and L. Zhigan, "Adaptive speed control for permanentmagnet synchronous motor system with variations of load inertia", IEEE Transactions on Industrial Electronics, vol. 56, no. 8, pp. 3050-3059, 2009.

[11] R, Taormina, "Artificial neural network simulation of hourly groundwater levels in a coastal aquifer system of the venice lagoon", Engineering Applications of Artificial Intelligence, vol. 25, no. 8, pp. 1670-1676. 2012.

[12] C.T. Cheng, "Long-term prediction of discharges in Manwan Reservoir using artificial neural network models", Lecture Notes in Computer Science, vol. 3498, pp. 1040-1045, 2005.

[13] K.W. Chau, "Application of a PSO-based neural network in analysis of outcomes of construction claims", Automation in Construction, vol. 16, no. 5, pp.642-646, 2007.

[14] W. Hongxin, H. Jun, and X.. Yongchun, "Characteristic modelbased all-coefficient adaptive control method and its applications," IEEE Transactions on Systems, Man, and Cybernetics, vol. 37, no. 2, pp. 213-220, 2007.

[15] W. Hongxin, H. Jun, and X. Yongchun. "Characteristic ModelBased Intelligent Adaptive Control, China Science and Technology Press: Beijing, 2009

[16] L. Jinkun, Sliding Mode Control Design and MATLAB Simulation. Tsinghua University Press: Beijing, 2012.

(C) Wang et al.; Licensee Bentham Open.

This is an open access article licensed under the terms of the Creative Commons Attribution Non-Commercial License (http://creativecommons.org/licenses/by-nc/3.0/) which permits unrestricted, non-commercial use, distribution and reproduction in any medium, provided the work is properly cited. 